\title{
Interpreting Quantum Entanglement: Steps towards Coherentist Quantum Mechanics
}

\section{Claudio Calosi and Matteo Morganti}

\begin{abstract}
We put forward a new, 'coherentist' account of quantum entanglement, according to which entangled systems are characterized by symmetric relations of ontological dependence among the component particles. We compare this coherentist viewpoint with the two most popular alternatives currently on offerstructuralism and holism-and argue that it is essentially different from, and preferable to, both. In the course of this article, we point out how coherentism might be extended beyond the case of entanglement and further articulated.
\end{abstract}

\section{Introduction}

2 Quantum Dependence

2.1 From entanglement to dependence

2.2 Relations of dependence

3 Structuralism and Holism

4 Against Structuralism

5 Against Holism

6 Coherentism

6.1 Coherentism: The account

6.2 Coherentism: The best of both worlds?

7 Conclusion

\section{Introduction}

We put forward a novel account of quantum entangled systems that departs substantially from those in the current literature. This account, we claim, makes sense of some important ideas that underpin extant ontological views of the quantum domain, while at the same time steering clear of some problematic commitments entailed by these views. In particular, the view that we present has it that entanglement should be understood in terms of the existence of symmetric non-mereological dependence relations, giving rise to 
what we will call 'coherentist' metaphysical structures analogous to the interconnected 'webs of beliefs' invoked by coherentist epistemologists. This entails a departure from a widespread view according to which nature comes structured in a hierarchy of vertical 'levels' ordered by dependence relations, and in which the nature of the 'more fundamental' determines the nature of the 'less fundamental'. Such hierarchical assumption is not abandoned by recent 'revisionary' attempts to interpret the evidence coming from the quantum domain. In particular, it is not abandoned by structuralists, who typically claim that reality is constituted by a vertically ordered sequence of physical relations that do not to depend on, and are instead more fundamental than (or at least as fundamental as) physical objects. Nor is it abandoned by holists of the monist variety, who claim that composite physical systems are such that the whole is ontologically prior to its parts. Dropping the hierarchy assumption, however, is both possible (in spite of a widespread contrary opinion, symmetric dependence relations entail no inconsistency) and, we claim, advisable. The plan of the article is as follows: In Sections 2-5, we argue that genuine entanglement gives rise to relations of ontological dependence, and critically assess the two main alternatives put forward in the recent literature in order to account for such relations: structuralism and holism. In Section 6, we put forward the coherentist account and discuss (some of) its theoretical virtues. A brief conclusion follows in Section 7 .

\section{Quantum Dependence}




\subsection{From entanglement to dependence}

Recently, a fairly widespread agreement has emerged on the fact that entanglement involves relations of dependence (see Esfeld and Lam [2011]; French [2010]; Schaffer [2010]; Wolff [2012]; McKenzie [2014]; Ismael and Schaffer [forthcoming]). Consider a paradigmatic example of entanglement, the singlet state:

$$
|\psi\rangle=|\uparrow\rangle_{1}|\downarrow\rangle_{2}-|\downarrow\rangle_{1}|\uparrow\rangle_{2}{ }^{1}
$$

As is well known, states like Equation (1) support considerations such as: 'if we measure spin up for the first particle we will necessarily measure spin down for the second', ${ }^{2}$ or 'if we had measured spin down for the first particle we would have obtained spin up for the second'. Both claims involve a modal element. Thus, entanglement can be said to correspond to certain modal connections holding between the entangled parts. Through the application of a weak, but plausible, variant of Hume's dictum (see Wilson [2010]), according to which there are no modal connections between independent entities, one obtains that the systems in question are not independent. It is the precise features of such dependence that we want to explore further in what follows.

An important remark is in order before continuing: a crucial distinction must be drawn between entanglement that arises purely

\footnotetext{
${ }^{1}$ We will always neglect normalization constants for simplicity.

${ }^{2}$ See (Ismael and Schaffer [forthcoming], Section 3.2). The necessity involved in the claim is, arguably, nomological necessity.
} 
because of the anti-symmetrization of a state of many particles of the same type, and genuine entanglement, not reducible to the previous type. This distinction originates from (Ghirardi et al. [2002]), and is adopted in (Ladyman et al. [2013]). ${ }^{3}$ To appreciate the distinction, consider a two-particle system in the state described in Equation (2):

$$
\left|\uparrow>_{1}\right| \downarrow>_{2} \cdot{ }^{4}
$$

Equation (2) does not capture all the degrees of freedom of the two particles. Let us then include another degree of freedom, the spatial degree of freedom, assuming that there are only two possible locations for particles 1 and 2 , represented by $\left|x_{1}\right\rangle$ and $\left|x_{2}\right\rangle$, respectively. Suppose we get Equation (3):

$$
\left|x_{1}>_{1}\right| \uparrow>_{1}\left|x_{2}>_{2}\right| \downarrow>_{2}
$$

The state described in Equation (3) is not anti-symmetrized. Antisymmetrization yields:

$$
\left|x_{1}>_{1}\right| \uparrow>_{1}\left|x_{2}>_{2}\right| \downarrow>_{2}-\left|x_{2}>_{1}\right| \downarrow>_{1}\left|x_{1}>_{2}\right| \uparrow>_{2} .
$$

As a matter of fact, Equation (4) cannot be written as a factorizable state. Thus, one may conclude, it is an entangled state. The problem

\footnotetext{
${ }^{3}$ Thanks to an anonymous referee for pressing us on this crucial issue.

${ }^{4}$ Our presentation follows closely (Ladyman et al. [2013], pp. 216-7). For more details, the interested reader is referred to the original publication, and to the more comprehensive (Ghirardi et al. [2002]).
} 
is that given that we started out from the state described in Equation (2) we do not expect any significant correlations between the spin of the two particles, hence no modal connections between them. And in fact, this is exactly what happens. The spin-probability distribution factorizes (Ladyman et al. [2013], p. 217). Thus, we should ensure that the state described in Equation (4) does not count as entangled in the relevant sense. ${ }^{5}$ This is achieved by maintaining that a system is genuinely entangled if and only if it gives rise to non-local correlations that violate some form of Bell-inequality. ${ }^{6}$ Ghirardi et al. ([2002], p. 61) propose a (provably equivalent) definition: a system is entangled if and only if it is not the case that the reduced density operator of each subsystem is a projection operator onto a onedimensional manifold. This entails that one or more of the subsystems cannot be associated to a unique, well-defined state vector, and be consequently regarded as possessing a complete set of objective properties of its own.

Let us then call the entanglement that merely arises from antisymmetrization but does not give raise to non-local correlation 'nonGMW entanglement'. Accordingly, let us call 'GMW entanglement' the entanglement that does give rise to non-local correlations violating some form of Bell inequality. It should be clear that GMWentanglement is the truly interesting form of entanglement and the

\footnotetext{
${ }^{5}$ That does not mean that this sort of entanglement is devoid of any physical meaning, just not the one we are after; see (Ladyman et al. [2013], pp. 217-8).

${ }^{6}$ Given an aptly defined quotient space on the tensor product space, called the wedge product space, it can be shown that a state is genuinely entangled if and only if the state cannot be written as a wedge-product of state vectors for the component particles. This ensures that the connection between non-factorizability (in the wedge-product space) and genuine entanglement is preserved.
} 
only relevant form in the present context. Only this entanglement supports actual, significant correlations, hence modal connections, between the entangled particles. Thus, we will restrict our attention to genuine entanglement, that is, GWM-entanglement.

\subsection{Relations of dependence}

Lately, philosophers of physics have turned their attention to different relations of 'ontological dependence'. Among others, French ([2010], [2014]), Wolff ([2012]), and McKenzie ([2014]) tried in particular to exploit the earlier work done on the logical and metaphysical status of ontological dependence relations (see Fine [1995]; Correia [2008]; Lowe and Tahko [2015]) with a view to providing a firm basis, or a critical assessment, of physical structuralism in the quantum domain. No doubt, this is a much welcome development. Yet some subtleties have been neglected and an important alternative framework ignored altogether.

Let us try to make some progress by taking an initial lead from Correia ([2008]), who distinguishes, first of all, between existential and essential dependence, ${ }^{7}$ and then identifies four different relations of essential dependence (in what follows, we use the sentential

\footnotetext{
${ }^{7}$ Essential dependence might raise some worries in the present context, for, as French ([2010], p. 106) puts it, 'essentialism has not typically been viewed all that favourably in the context of modern physics'. Nonetheless, there is widespread agreement on the idea that we should indeed allow ourselves essentialist talk, especially because 'essence too may be relatively innocuous [...] It may turn out, in fact, that what Fine has in mind by 'essence' is just the sort of thing that we're used to dealing with all the time in particle physics' (McKenzie [2014], p. 8). In line with the literature, then, we will use the essentialist framework here, without making any heavy commitment to essences and/or to particular views concerning their nature, and merely with a view to picking out the relevant features of certain (kinds of) objects.
} 
operator $\square_{x}$ for ' $x$ is essentially such that' and $E$ for the 'existence predicate') $)^{8}$ :

Rigid Essential Involvement: For some $R$,<smiles>[Te][Te]</smiles>

(for some relation $R, x$ is essentially related to $y$ by $R$ ).

Rigid Essential Necessitation:

$\square_{x}(E x \rightarrow E y)$

( $x$ is essentially such that it exists only if $y$ exists).

Generic Essential Involvement: For some $R$,

$$
\square_{x} \exists y(F y \wedge R x y)
$$

\footnotetext{
${ }^{8}$ It should be noted that one may endorse reductionism, that is, accept the biconditional $\square_{x} \phi(x) \leftrightarrow \square(E x \rightarrow \phi(x))$. According to reductionism, an essential property is just a property that something has in every possible world in which it exists. In other words, it is a purely modal account of essences and essential properties. However, well-known arguments against reductionism should be taken into account. Fortunately, we do not have to take a stance on this here. Note, in connection to this, that the existence predicate may (or may not, depending on the endorsement or rejection of the quantificational view of existence) be defined via $E x=_{d f} \exists y(x=y)$.
} 
(for some relation $R, x$ is essentially related to an object that is $F$ by $R)$.

Generic Essential Necessitation:

$$
\square_{x}(E x \rightarrow \exists y(F y))
$$

( $x$ is essentially such that it exists only if something is an $F$ ).

In all these cases, $x$ is the dependent entity whereas $y$ is the dependee, so that $x$ ontologically depends on $y$. The thought is that something depends on something else if and only if the existence of the latter (as the very entity it is) is a necessary condition for the existence of the former (as the very entity it is). ${ }^{9}$ Essentialist talk should thus be understood in terms of the existence and distinctive properties of the entity or entities in question.

\footnotetext{
${ }^{9}$ A caveat is in order. Fine ([1995], p. 276) distinguishes between constitutive essence and consequential essence: 'A property belongs to the constitutive essence of an object if it is not had in virtue of being a logical consequence of some more basic essential properties; and a property might be said to belong to the consequential essence of an object if it is a logical consequence of properties that belong to the constitutive essence'. This notion of consequential essence is intuitive but problematic. Consider the property of being either identical to or distinct from $x$. This property is part of the consequential essence of everything. This in turn will yield that everything depends on everything. To avoid this consequence Fine requires that $x$ depends on $y$ if and only if $y$ cannot be 'generalized out' of the consequential essence of $x$. In what follows, we will take for granted that all the interesting claims of dependence have passed this 'generalization' test.
} 
Necessitation rather than involvement appears to be the preferred option in the literature. French ([2010], p. 100), for instance, discusses various options and ends up endorsing the following

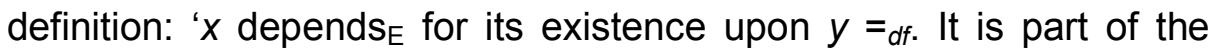
essence of $x$ that $x$ exists only if $y$ exists', which is basically Equation (6). Similarly, McKenzie ([2014]) takes Equation (6) the be the most general form of any dependence claim, and the most useful in the context of structuralism in quantum physics. ${ }^{10}$ We will follow suit.

A qualification is required, though. It is not so much existence per se that is at stake, but rather the existence of something with a specific set of properties. While two or more entangled particles may well have existed without becoming entangled, it is always the case that as soon as such particles enter into genuine entanglement, they do not have independent complete sets of objective properties, and instead depend on one another with respect to their qualitative profiles. ${ }^{11}$ Or at least, this is what we will argue for. Thus, from now on, unless otherwise specified, we will assume that ontological

\footnotetext{
${ }^{10}$ Wolff ([2012]) uses instead the notion of identity dependence (see Tahko and Lowe [2015]), which is derivable from an instance of Equation (5).

11 Of course, what exactly 'qualitative profile' refers to should be specified, especially whether it includes all properties or only some of them. In the latter case, presumably the distinction between accidental and essential properties should also be considered. Here, however, it is sufficient to assume that the entities in question are mutually dependent with respect to some properties, specified on the basis of the relevant physics. In particular, as we will see, physical systems can be entangled in some degrees of freedom and not others, and only entangled degrees of freedom track the dependence relations we are presently interested in. Further details on the issue of qualitative profiles will have to wait for another occasion.
} 
dependence is the relation whereby something $x$ depends on something $y$ for its existence and its properties. ${ }^{12}$

Bearing this in mind, suitable generalizations of the previous schemas also need to be considered, so as to allow for different objects or pluralities of objects depending on different other objects or pluralities. The most general cases, restricting our attention to necessitation, are the following two:

Collective Rigid Essential Necessitation:

$$
\square_{x x}\left(E x_{1} \wedge \ldots \wedge E x_{n} \rightarrow E y_{1} \wedge \ldots \wedge E y_{m}\right)
$$

$\left(x_{1}, \ldots, x_{n}\right.$ are essentially collectively such that they exist only if $y_{1} \ldots, y_{m}$ exist, where $x x$ is a plural variable for the plurality containing $\left.x_{1}, \ldots, x_{n}\right) .^{13}$

Collective Generic Essential Necessitation:

$$
\square_{x x}\left(E x_{1} \wedge \ldots \wedge E x_{n} \rightarrow \exists y y(F y y)\right)
$$

\footnotetext{
12 In other words, the claim is one of dependence in a specific respect. Using the grounding terminology for just a moment, the claim is not, say, 'a grounds $b$ ', but rather 'the fact that a exists and possesses features $P, Q, \ldots, S$ grounds the fact that $b$ exists and possesses features $W, X, \ldots, Z^{\prime}$, where $P, Q \ldots, X$, and $Z$ stand for the qualitative profile of $a$ and $b$.

${ }^{13}$ We are assuming that a plurality $x x$ exists if and only if all of its members $x_{1}$, $\ldots, x_{n}$ exist. Also notice that Equation (9) is normally regarded as distributive on the right, as equivalent to the conjunction of all statements of the form 'It's part of the essence of $x_{1}, \ldots, x_{n}$ that $E x_{1} \& \ldots . \& E x_{n} \rightarrow E y_{i}^{\prime}$ for every $i$ between 1 and $m$.
} 
$\left(x_{1}, \ldots, x_{n}\right.$ are essentially collectively such that they exist if only some Fs exist).

It should be noted that collective dependence relations may carry significantly less information about the nature or the essence of individual members of the plurality in question than their singular counterparts. This is especially so if such dependence is genuine and irreducible, that is, not analysable in terms of dependence relations having singular entities in the dependent place. This will have a bearing on our later critical assessment of the relevant ontological alternatives. Having said this, let us now look more specifically at quantum systems and the sort of dependence relations they (may) exhibit.

\section{Structuralism and Holism}

There are two main candidates currently on offer for understanding the metaphysical nature of quantum systems, especially in the case

of entanglement. We will refer to them as structuralism (French [2010]; Esfeld and Lam [2011]; Wolff [2012]; McKenzie [2014]) and holism (Schaffer [2010]; Schaffer [2014]; Ismael and Schaffer [forthcoming]), respectively.

A structuralist understanding of quantum entanglement is typically but a plank in the larger programme of ontic structural realism (OSR). According to one of the main advocates of OSR, James Ladyman ([2014], p. 23), 'Any form of structural realism based 
on an ontological or metaphysical thesis that inflates the ontological priority of structure and relations' qualifies as OSR. As a typical example of this, symmetric yet irreflexive physical relations are invoked by some philosophers in order to ensure the weak discernibility of quantum particles. ${ }^{14}$ And structuralists typically claim that these relations are fundamental, and such that the weakly discernible objects depend on them. For the sake of simplicity, consider the state described in Equation (1). In that case, the two particles in the singlet state are anti-correlated, meaning that upon measurement one necessarily obtains opposite values for their statedependent observables, such as spin along a particular direction. In terms of dependence, the hallmark of the structuralist understanding of these physical systems is then the following:

Structuralism:

$$
\square_{x x=\left\{x_{1}, x_{2}\right\}}\left(E x_{1} \wedge E x_{2} \rightarrow \exists R^{i r r, s y m}\left(R x_{1} x_{2}\right)\right) .^{15}
$$

Fermions 1 and 2, that is, are taken to depend on the existence of the irreflexive symmetric relation 'having opposite spin to'. Crucially, such relation is regarded by structuralists as a physical entity that is

\footnotetext{
14 Following (Saunders [2006]; Saunders and Muller [2008]; Muller and Seevinck [2009]). For an up-to-date critical discussion, see (Bigaj [2015]). To be clear, weak discernibility is neither necessary nor sufficient for entanglement, which is our main focus here. We are just using it as an example based on a theme that has been hotly debated in the recent literature and is often used as a basis for structuralist interpretations of the quantum domain.

${ }^{15}$ This is basically what McKenzie ([2014]) ends up with.
} 
ontologically prior to the entangled particles ${ }^{16}$. This means that (entangled) quantum objects depend, or even are reducible to, ${ }^{17}$ more fundamental physical relations. ${ }^{18}$

The other relevant understanding of quantum entanglement is, as mentioned, holism (Schaffer [2010], [2014]; Ismael and Schaffer [forthcoming]). Focus again on the two-particle entanglement case: the holist claims that there is a quantum whole that has the two subsystems as parts, and that the sub-systems depend on such a whole, which is ontologically prior. The basic argument in favour of such an understanding is essentially the following: Neglecting particular interpretations of quantum mechanics, ${ }^{19}$ it is always the case that the state of the whole uniquely determines that of the parts, whereas the converse does not hold-entanglement being, of course, the paradigmatic counterexample. According to the holist, the best explanation for this is that, in an ontological sense, the whole comes first. Employing the formal resources that we have used so far, we can formulate this as the following thesis:

Holism:

\footnotetext{
${ }^{16}$ Or ontologically on a par with, as contended by 'moderate' ontic structural realists; see (Esfeld and Lam [2008]).

${ }^{17}$ See (Wolff [2012]) for scepticism about reduction.

${ }^{18}$ More physically realistic examples are 'having a value different from zero for the commutator of momentum (of one particle) and position (of another particle)' or, more generally, "having a non-zero value for the variance of some quantity computed on the two particles'. The discussion of the criteria for identifying the 'right' discerning relations is still ongoing, however. Having said this, the exact nature of discerning relations is irrelevant for our main argument. Thus, we will stick to 'opposite spin' for the sake of simplicity.

${ }_{19}$ In particular, (some of) the modal interpretations, which seem to have somewhat different consequences; see (Calosi [2017]).
} 


$$
\square_{x x=\left\{x_{1}, x_{2}\right\}}\left(E x_{1} \wedge E x_{2} \rightarrow \exists y\left(x_{1}<<y \wedge x_{2}<<y\right)\right),
$$

where $<<$ is the mereological notion of proper parthood. ${ }^{20}$

Notice that Equation (12) does not mention properties explicitly, and just states that the existence of something renders the existence of something else necessary. However, as pointed out earlier, this can, and should, be understood in terms of existence plus properties, as the claim that something exists (in the way it does), thanks to the fact that something else exists (in the way it does)-where the way something exists corresponds to that something's qualitative profile. Indeed, the basic holist argument just mentioned explicitly trades on qualitative profiles, insofar as it focuses on the quantum states of wholes and parts. ${ }^{21}$

Importantly, while physical wholes can be 'local', in the quantum domain holism as a claim of ontological priority naturally leads to stronger monist views. For instance, Ismael and Schaffer ([forthcoming]) argue that the most relevant whole comprises everything physical, that is, it is identical with physical reality as a whole. The fundamental 'one' may be identified, they suggest, either with the largest object existing in space and time or, alternatively,

\footnotetext{
${ }^{20}$ As per the standard definition, where parthood is taken as primitive: $x$ is a proper part of $y$ if and only if $x$ is part of $y$ and $x$ is distinct from $y$.

${ }^{21}$ Here 'qualitative profile' is to be understood loosely, so as to include identity conditions when relevant. On the notion of 'qualitative profile', see also Footnote 11.
} 
with the wave-function as an entity existing in $3 n$ configuration space ( $n$ being the relevant number of 'particles' or, more broadly, 'components'). ${ }^{22}$ Distinguishing holism from this stronger claim is essential: as we will see, the shift from holism to monism is an unwelcome consequence of the view-while holistic elements broadly understood may well be, and in fact should, be part of a plausible interpretation of quantum entanglement.

We will now provide a critique of both structuralism and holism as they have been presented in the literature. To be clear, the arguments put forward are not intended to be knock-down arguments against these positions. Rather, they are meant to highlight difficulties that we believe have not yet been appreciated (at least not fully), but are serious enough to motivate the search for an alternative account.

\section{Against Structuralism}

The problem with structuralism, we contend, is twofold: (i) it depends on a rather substantive and controversial reading of the quantum state in relational terms, and (ii) it is less informative than what one might expect insofar as it amounts to a claim of collective generic dependence. $^{23}$

\footnotetext{
${ }^{22}$ It is worth pointing out that structuralism and monistic holism are not exclusive options. French ([2014]), for instance, takes a specific form of monism whereby the only existing thing is an all-encompassing structure as the best metaphysical framework for physical structuralism (a case of 'existence-monism', rather than the Schaffer-style 'priority-monism', is discussed in Section 5).

${ }^{23}$ McKenzie ([2014], p. 8) is explicit in admitting this is just generic dependence.
} 
Let us start with the first charge. Consider the simple singlet state. As mentioned, structuralists who endorse Equation (11) give a relational reading of the state according to which the relevant quantum systems depend on the irreflexive and symmetric relation of 'having opposite spin'. However, first, if the claim of ontological priority is to be upheld a non-extensional definition of the relations in question needs to be provided. But it appears very hard to formulate one (this is the basic idea, we take it, in Wolff [2012]; McKenzie [2014]). Second, and crucially, it is in fact by no means obvious that one is dealing with relations in the first place.

Consider, for instance, entangled fermions. The Pauli exclusion principle states, roughly, that no two fermions in a given system can have exactly the same values for all quantum numbers. ${ }^{24}$ That is, two fermions in the same system will always have incompatible determinate values for at least a quantum number $Q$. Now, the first thing to notice is that if this were sufficient for concluding that there are two related entities in the system, then the two fermions would in fact be told apart from each other also by the following symmetric yet irreflexive relation: $R^{*}=$ 'having incompatible values for quantum number Q'. Indeed, the relation of 'having opposite spin' is but an example of $R^{\star}$ where $Q$ is the spin quantum number. At any rate, independent of the specific properties one focuses on, the key question is: is $R^{*}$ a genuine physical relation? As a matter of fact, it looks as though, while relations such as this can be very easily

\footnotetext{
${ }^{24}$ Pauli ([1925]) writes that 'there can never be two or more equivalent electrons in an atom, for which in strong fields the values of all quantum numbers $n, I, j$, and $m$ are the same'.
} 
reconstructed from the theory at the linguistic/formal level, ontologically it is far from obvious that one is in fact dealing with actual relations, and taking this for granted essentially assumes what must be demonstrated. Note that this sort of consideration is different from the often-heard circularity charge. It does not concern the possibility of relations that do not presuppose, hence depend on, their relata (which we do not complain about); instead, it has to do with what we are (or are not) allowed to infer from the theory at the ontological level. Following, for instance, Dieks and Lubberdink ([2011]) and Jaeger ([2010]), one may think that there are reasons for not connecting particle labels and individual systems in a one-toone fashion-the separate Hilbert spaces that enter a tensor product on the one hand, and putatively separate physical systems described by each Hilbert space on the other. ${ }^{25}$ And if there is no such direct connection, it becomes plausible to also think that facts such as those concerning fermions and the exclusion principle may not point to an ontology of relations and structures, and leave instead the metaphysical question open.

Let us now move on to the second charge. Insofar as the irreflexive and symmetric relation crucially weakly discerns the two particle tokens in the singlet state, the structuralist might want to

\footnotetext{
25 These reasons have to do, roughly, with the fact that particle labels can be taken to correspond to specific physical systems only in the limiting case in which the relevant wave-function is 'concentrated'. But this amounts to assuming the classical limit, hence the very ontology of well-defined distinct objects that cannot be transferred to quantum mechanics without argument.
} 
claim that the particles depend individually on such a relation. One may try to cash this out in two ways ${ }^{26}$ :

$$
\begin{aligned}
& \square_{x_{1}}\left(E x_{1} \wedge E x_{2} \rightarrow \exists R^{i r r, s y m}\left(R x_{1} x_{2}\right)\right), \\
& \square_{x_{1}}\left(E x_{1} \rightarrow E x_{2} \wedge \exists R^{i r r, s y m}\left(R x_{1} x_{2}\right)\right) .
\end{aligned}
$$

Both options are problematic, though. In the first case, Equation (13), we would have that something about the essence of $x_{1}$ and $x_{2}$ 'flows from the nature' of just $x_{1}$, to use Fine's suggestive expression. In the second case, Equation (14), $x_{1}$ would depend not just on the existence of a relation but also on another of its relata, namely, $x_{2}$. Either way, this is contrary to the spirit of structuralism, which claims that objects depend on structures/relations, not some other objects/relata. To make progress, it seems, it is necessary to focus on collective essences instead, and emphasize the fact that a collective essence may not correspond to separate individual essences. The standard Finean example is as follows:

$\square_{S, E T}($ Socrates $\neq$ Eiffel Tower $)$,

$\square \square$ S (Socrates $\neq$ Eiffel Tower $),$

$\square \square_{E T}$ (Socrates $\neq$ Eiffel Tower).

\footnotetext{
${ }^{26}$ The choice of $x_{1}$ is entirely arbitrary; the same goes for $x_{2}$.
} 
The thought is that whereas it is essential to Socrates and the Eiffel Tower collectively that they are distinct, it is not essential to Socrates to be distinct from the Eiffel Tower insofar as he is not essentially related to the Eiffel Tower in any way. And the same goes, mutatis mutandis, for the Eiffel Tower. ${ }^{27}$ Thus, the structuralist is forced to concede that despite the fact that the physical relation in Equation (11) weakly discerns the individual systems, the dependence from such a relation is not individual dependence, but rather a collective one. That is, the relation in question (weakly) discerns the particles individually, but the latter only collectively depend on it. This may not be a problem in itself, especially for those structuralists who either eliminate objects or conceive of them as less than individuals in some sense (sometimes the term 'relational' is used). However, an actual difficulty emerges when it comes to the specific nature of the collective dependence relation in play.

${ }^{27}$ Someone who endorses reductionism as defined in Footnote 8 might claim that the only relevant way for Equation (15) to be true, and Equation (16) to be true as well is for there to be a world in which Socrates exists, but the Eiffel Tower does not. In this case, however, we should not say that Equation (15) is false. Rather, we should say that it is meaningless, insofar as the second term is an empty term. On this reductionist construal, far from being significantly different, Equations (13) and (11) would turn out to be logically equivalent. Fine's response, we take it, would be that he rejects reductionism. Without necessarily following Fine on this, we just note here that reductionism obfuscates exactly the key difference that we are pointing at, namely, the difference between (a) the case in which, necessarily, the existence of two things has a certain consequence in virtue of the essence of only one of them, and (b) the case in which the same happens, but in virtue of the essence of the two things together. The point that there might be essential statements about collections that do not factor, so to speak, into essential statements about the individuals involved in the collection is made, to our minds convincingly, by Zylstra ([forthcoming]). 
While in some cases the structuralist can claim that $x_{1}$ and $x_{2}$ depend rigidly on relations such as 'having opposite spin', this is not generally the case, due to the fact that particles can be entangled in more than one degree of freedom. To illustrate this, let us consider a toy example. Consider the state described in Equation (1) once again. This is, to recall:

$$
|\psi\rangle=\left(|\uparrow\rangle_{1}|\downarrow\rangle_{2}-|\downarrow\rangle_{1}|\uparrow\rangle_{2}\right)
$$

Now, define the following vectors ${ }^{28}$ :

$$
\begin{aligned}
& \left|x_{1}\right\rangle=\frac{1}{\sqrt{2}}(|\uparrow\rangle+|\downarrow\rangle) \\
& \left|x_{2}\right\rangle=\frac{1}{\sqrt{2}}(|\uparrow\rangle-|\downarrow\rangle) \\
& |\uparrow\rangle=\frac{1}{\sqrt{2}}\left(\left|x_{1}\right\rangle+\left|x_{2}\right\rangle\right) \\
& |\downarrow\rangle=\frac{1}{\sqrt{2}}\left(\left|x_{1}\right\rangle-\left|x_{2}\right\rangle\right)
\end{aligned}
$$

Where $\left|x_{1}\right\rangle$ is the state of being located at $(0,0,0)$ and $\left|x_{2}\right\rangle$ the state of being located at $(1,0,0) .{ }^{29}$ It is easily checked that Equation (1) can be written as:

\footnotetext{
${ }^{28}$ Note that it is unphysical to define eigenstates of position as superpositions of spin eigenstates and vice versa. We only work with spin and position observables in order to make our point more perspicuous. A physically accurate example to make the same point would involve, for instance, spin components in different directions.

${ }^{29}$ In a suitable frame of Cartesian coordinates.
} 


$$
|\psi\rangle=\left(\left|x_{1}\right\rangle_{1}\left|x_{2}\right\rangle_{2}-\left|x_{2}\right\rangle_{1}\left|x_{1}\right\rangle_{2}\right)
$$

In this toy example, systems 1 and 2 are entangled both in the spin and in the position-degree of freedom. In particular they stand in the symmetric yet irreflexive relations of 'having opposite spin' and 'being one metre apart along the $x$-axis'. The crucial point is that it seems quite arbitrary to choose one relation over the other, hence the structuralist can at most make a claim of generic dependence. Generic dependence, however, is less stringent and informative than rigid dependence: is the spin correlation the ontologically basic item? Is it the distance relation? Is it both? Is it some third relation different from them? If we are correct that the dependence exhibited by quantum systems is necessarily generic and collective, no precise answer to this question can be given. From which it follows that structuralism is not a precise ontological thesis, in the sense that structuralists must take entangled relations as ontologically basic physical entities, but at the same time accept that these relations cannot be analysed any further in terms of physical observables-in terms of the actual properties of quantum systems.

One might object that our argument establishes a multiplicity of rigid dependencies rather than a generic dependence. ${ }^{30}$ This is a sensible reaction. However, it is up to the structuralist to come up with a full-fledged proposal able to account for such a multiple dependence. Here, we just point out that whenever systems are

\footnotetext{
${ }^{30}$ Thanks to an anonymous referee here.
} 
entangled in more than one degree of freedom, there may well be more than one rigid dependence relation involved on top of the generic one; however, the generic and the rigid relations may well differ both in content and adicity. ${ }^{31}$ This, we think, would detract from the uniformity of the account. On top of this, it would remain the case that faced with an undeniable proliferation of allegedly basic entities, structuralists would still owe us an account of which relations are here truly fundamental: the generic one, any of the rigid ones? All the rigid ones? All the rigid ones and the generic one?

Perhaps a fully-fledged structuralist account of entangled systems can be provided, but for the time being it is fair to point out that simply claiming that 'entanglement relations are fundamental' is, contrary to what appears to be normally assumed in the literature, insufficient. For, entanglement is not so much a physical property of quantum systems, as a way for certain observables to be exemplified by such systems. However, in order for structuralism to be a truly compelling, science-based position, we need to be told what exactly the physical content of the relevant relations is, which observables the correlated quantum objects depend on. In view of this, it seems fair to claim that structuralism needs, to say the least, a good amount of further work to become a truly compelling view of the nature of quantum systems. $^{32}$

${ }^{31}$ For example, in the case we discuss in Footnote 40, the generic relation would be a three-place relation, whereas the two rigid ones will be two-place relations.

${ }^{32}$ Notice that our argument didn't even refer to the key issue, nicely pointed out by (McKenzie [forthcoming]), that a structuralist reduction of all properties seems in fact hard to carry out, as at least some state-independent properties are left 


\section{Against Holism}

Moving now on to holism, the problem with it is analogous to that concerning structuralism. On the one hand, holism rests upon a controversial assumption about what the quantum state is supposed to represent. On the other hand, either (i) it sticks to a generic notion of dependence, thus becoming uninformative, or (ii) every attempt to turn the generic clam into a rigid claim of dependence will fail to provide a satisfactory account of some subtle features of entanglement.

Starting from the first point, the holist has, in a sense, the opposite problem with respect to that faced by the structuralist. That is, she has to argue that all facts concerning the parts depend on facts about the whole, hence that all fundamental properties are monadic properties of the whole itself (be these traditional properties such as 'having total spin of zero' or more complicated properties such as 'being bosonic here' or 'being two-fermions-one-metre-apartish there'). The holist may argue (see Schaffer [2010], [2014]) that no matter how 'unusual' and/or under-determined the relevant properties may be, this reading of the quantum state warrants a high degree of uniformity, and should consequently be preferred-even at the cost of not attributing seemingly fundamental properties such as, say, the minimal amount of electric charge to what intuitively would look as their natural bearers, such as point-like particles.

underdetermined by the typical group-theoretic considerations invoked by structuralists in the quantum context. 
In connection to this, in particular, the idea has been put forward (see, for example, Schaffer [2010]) that holism allows one to postulate the same monadic property (such as 'being entangled') for systems with different numbers of components, whereas a reading in terms of relations between parts forces one to admit different relations of different adicity for systems with different number of components. This argument, however, is far from uncontroversial, as witnessed by the objections raised in (Morganti [2009a]; Bohn [2012]; Calosi [2014]). For one, why think that the pluralist needs different relations with different fixed adicity and not a single multi-grade relation?

As for the claim that all properties are monadic properties of the whole, it appears at odds with at least some physical facts. Consider, for instance, a state like that in Equation (19), and the spatial degree of freedom. The relational reading of such a state sounds pretty straightforward: it just says that quantum systems 1 and 2 stand in the relation of 'being one metre apart along the $x$-axis' from each other. On the other hand, the holist reading seems less satisfactory, for it should say something like 'the whole is one metre long, and this is prior to the existence of two parts that are one metre apart from each other', which is far from transparent.

Consider next four particles $1,2,3,4$ that can occupy only four positions corresponding to vectors $\left|x_{1}\right\rangle,\left|x_{2}\right\rangle,\left|x_{3}\right\rangle,\left|x_{4}\right\rangle$, such that: (i) $\left|x_{1}\right\rangle$ is the state of being located at $(0,0,0)$, (ii) $\left|x_{4}\right\rangle$ the state of being 
located at $(1,0,0)$; (iii) $\left|x_{2}\right\rangle,\left|x_{3}\right\rangle$ are states of being located between positions $\left|x_{1}\right\rangle$ and $\left|x_{4}\right\rangle$. Let the state of this four-particle system be ${ }^{33}$ :

$$
\begin{aligned}
& |\omega\rangle=\left|x_{1}\right\rangle_{1}\left|x_{2}\right\rangle_{2}\left|x_{3}\right\rangle_{3}\left|x_{4}\right\rangle_{4} \\
& -\left|x_{1}\right\rangle_{4}\left|x_{2}\right\rangle_{3}\left|x_{3}\right\rangle_{2}\left|x_{4}\right\rangle_{1}
\end{aligned}
$$

If the only thing the holist can say is that Equation (20) attributes 'having a one-metre extension' to the mereological sum of 1, 2, 3, 4 (let's call it '1234'), she is losing precious information. Certainly, the holist might reply that she can also attribute to 1234 the properties of having four parts, and having parts that are related in a certain way (for example, excluding that 1 and 4 are ever in between 2 and 3 ). But since she is forced to mention particular relations between specific parts, and all the available information about 1234 can be given in terms of these, she owes us an account of why these relations and parts are not enough at the ontological level. ${ }^{34}$

Moving now on to the kind of dependence that is called into play, things might seem brighter in this respect for the holist than for the structuralist. For, there seem to be natural candidates for formulating a rigid dependence claim. Consider the following:

\section{Holism*:}

\footnotetext{
${ }^{33}$ We are neglecting anti-symmetrization for simplicity.

${ }^{34}$ Notice, in particular, that once relations are also taken into account the idea that the whole contains more information than its parts becomes less compelling, at least on a mereological understanding of properties and relations. This is exactly the reason why structuralists account for the 'emergent' nature of quantum entanglement in terms of entangled particles plus irreducible correlations.
} 
$\square_{x x=\left\{x_{1}, x_{2}\right\}}\left(E x_{1} \wedge E x_{2} \rightarrow E\left(x_{1}+x_{2}\right)\right)$

where + stands for mereological sum.

Under any extensional mereology, $x_{1}+x_{2}$ is unique, so that Equation (21) is a truly rigid claim of dependence, pointing to the specific composite system that is, crucially, the only relevant whole in the case at hand. Despite its attractiveness, however, this understanding of the situation faces a number of problems.

First, entanglement, via modal connections, tracks dependence not composition. In fact, there are (i) cases in which we have entangled degrees of freedom within a single particle, and (ii) cases in which non-classical correlations emerge in decomposable systems, systems that may just be regarded as collections of independent, simpler systems (this is known as 'quantum discord'). ${ }^{35}$ Thus, the connection between entanglement and composition is far from straightforward. As a matter of fact, that there is always a (uniquely defined) mereological sum in cases like Equation (21), and everything significant there is to say about quantum entanglement is always in terms of (uniquely defined) mereological wholes are instead very substantial claims, definitely in need of further support. ${ }^{36}$

\footnotetext{
${ }^{35}$ See (Hasegawa [2012]) on point (i), and (Earman [2015]; Li and Luo [2008]) on (ii).

${ }^{36}$ One may opt for a non-mereological understanding of the relevant wholes, for instance endorsing realism about the wave-function in configuration space and noticing the obvious fact that actual physical systems are in no way parts of the wave-function. However, this does not mitigate the problem, as the holist now
} 
A possible reply on behalf of the holist is that a suitably powerful mereology does the job. Schaffer, for instance, explicitly endorses unrestricted composition, which obviously guarantees that one can always identify a mereological whole corresponding to the relevant quantum state, even independently of the number of components and whether or not entanglement occurs. On the other hand, this is again a claim that has been contested in the literature. Caulton ([2015]), for instance, argues that fermionic composition provides counter-examples to the unrestricted composition axiom. ${ }^{37}$

Even granting that mereology is applicable in the quantum domain so as to support holism, there is another problem with Equation (21). To illustrate it, we need to introduce the distinction between maximally, or completely, entangled and non-maximally entangled states (see, among others, Horodecki et al. [2009], pp. 889-90; Ghirardi et al. [2002], p. 68). Let us say that a $n$-partite state is completely unentangled if and only if each component system 1 , $\ldots, n$ has its own pure state. A state is GMW-entangled-that is, in our earlier terminology, 'genuinely' entangled—if it is not completely unentangled. A GWM-entangled state is completely, or maximally, entangled if and only if the only system that is not decomposable in a non-entangled state is the total system $12 \ldots n$. Equivalently, only the total system can be assigned a pure state. Note now that there might

owes us a story concerning the connection between the whole in configuration space and the local objects, properties and facts that we experience-which is, of course, a well-known issue for wave-function realists.

37 More precisely, Caulton provides counterexamples also to finite-binary arbitrary fusions. Needless to say, this is problematic for the holist. Healey ([2013]) also questions the application of traditional notions of composition in contemporary physics. 
be GMW-entangled systems that are not maximally entangled, whose states are of the form:

$$
|\psi\rangle_{1 \ldots n}=|\psi\rangle_{1 \ldots i} \otimes|\psi\rangle_{i+i \ldots n}
$$

In the two-particle case the distinction between general (GMW)entanglement and maximal entanglement collapses. Yet this is not normally the case, for we might find states such as

$$
|\psi\rangle_{1234}=|\uparrow\rangle_{1}|\downarrow\rangle_{2}-|\downarrow\rangle_{1}|\uparrow\rangle_{2} \otimes|\uparrow\rangle_{3}|\downarrow\rangle_{4}-|\downarrow\rangle_{3}|\uparrow\rangle_{4},
$$

which are entangled insofar as $|\psi\rangle_{1234} \neq|\psi\rangle_{1} \otimes|\psi\rangle_{2} \otimes|\psi\rangle_{3} \otimes|\psi\rangle_{4}$ but are not maximally or completely entangled because $|\psi\rangle_{1234}=|\psi\rangle_{12} \otimes|\psi\rangle_{34}$.

Now, consider a maximally entangled four-particle system. There seems to be a clear sense in which the truly fundamental whole is 1234 , for the state of each part of 1234,12 included, will be fixed only by 1234 . This is exactly the kind of argument the holist put forward in the first place. However, this seems to run afoul of Equation (21), for Equation (21) entails that 1 depends rigidly on 12, not on 1234. Of course, the holist could stick to the claim that what is truly fundamental is 1234 -for instance, in virtue of the transitivity of ontological dependence. Consequently, the holist might argue that (i) the rigid dependence of each part on the 'minimal' whole-the whole that comprises those parts and nothing else-_counts less' than (ii) its 
rigid dependence on the 'larger' whole, which is the truly fundamental entity. But what would be the ground for this, that is, for determining 'true fundamentality', exactly? Of course, the holist cannot just assume the truth of his/her Parmenidean view of reality. If the criterion we are looking for is based on physical facts plus rigid dependence, we have (at least) two options to choose from, and only one of them can be kosher for the holist. Yet, the choice seems to be entirely underdetermined.

Further empirical evidence can be invoked that pulls in the same direction. Recently, it has been shown that it is possible to take a $n$-particle system in which all particles are only partly entangled and concentrate entanglement into a smaller number of maximally entangled parts, leaving the others unentangled (Bennett et al. [2006]). This process is called entanglement distillation. ${ }^{38}$ Conversely, one can spread entanglement over a large number of particles so that the initial total entanglement is preserved, and yet the particles are only partially entangled. This process is known as entanglement dilution.

The problem for the holist is that in these cases it appears natural to say that dependence relations hold just between the parts that are entangled (and their fusion, if there is such a thing), and there are no modal connections between the parts that, say, are left unentangled by the distillation process. Yet, physically, one still has a $n$-particle

\footnotetext{
${ }^{38}$ Or entanglement concentration, or entanglement purification.
} 
whole after the process. ${ }^{39,40}$ Suppose, however, that the holist insists on 'going large', and consequently suggests the following refinement of the view:

Holism**:

$$
\square_{x x=\left\{x_{1}, \ldots, x_{n}\right\}}\left(E x_{1} \wedge \ldots \wedge E x_{n} \rightarrow\left(E y\left(x_{1}<<y \wedge \ldots \wedge x_{n}<<y\right) \wedge \square \exists z(y<<z)\right)\right)
$$

Whereas Equation (21) says that the parts depend on the minimal whole, Equation (24) says that they depend on a maximal whole (a whole that has them as parts and is not part of anything else). Besides the fact that this move seems to be made for entirely a priori reasons, the problem with this is that the only way to turn Equation

\footnotetext{
${ }^{39}$ The connection should be clear: entanglement distillation basically turns nonmaximally entangled $n$-particles states into maximally entangled $m<n$-particle states; see (Wang et al. [2012]).

${ }^{40}$ Here is another interesting argument based on an example, which we borrow from (Bigaj [2012]) with only a slight change of notation (we are grateful to an anonymous referee for the pointer). Consider a three-particle system 123, whose component parts, 1, 2, and 3 are associated with four-dimensional Hilbert spaces spanned by vectors $\left.|+\rangle,|->,|^{*}\right\rangle$, and $|\#\rangle$. The state of 123 is $\left.|\Psi\rangle_{123}=\left.|+\rangle_{1}|-\rangle_{2}\right|^{*}\right\rangle_{3}+$ $\left.\left.\left|+>_{1}\right| \#>_{2}\left|+>_{3}+\right|->_{1}\left|+>_{2}\right|^{*}\right\rangle_{3}+\left|->_{1}\right|^{*}\right\rangle_{2}|+\rangle_{3}$. It is possible to prove that this state is maximally entangled and yet the state of 13 is a product state. This suggests that system 1 is entangled with system 2, system 2 is entangled with system 3 , but system 1 is not entangled with system 3 . Bigaj suggests an interesting physical interpretation. Let $\left.|+\rangle=\left|x_{1}\right\rangle|\uparrow\rangle ;|-\rangle=\left|x_{1}\right\rangle|\downarrow\rangle ;\left.\right|^{*}\right\rangle=\left|x_{2}\right\rangle|\uparrow\rangle$, and $|\#\rangle=\left|x_{2}\right\rangle|\downarrow\rangle$, where $\left|x_{1}\right\rangle$ and $\mid x_{2}>$ represent the only two possible locations of the particles. Then 1 is entangled with 2 in the spin degree of freedom, whereas particle 2 is entangled with 3 in the position degree of freedom. What is the most plausible holist interpretation of such a state? According to the holist the most fundamental physical system is 123. But this analysis clearly misses precious quantum information about correlations between the parts of the whole system. It looks as though 123 has two parts ( 1 and 2 ) that exhibit spin-correlation supporting certain modal connections, and two parts (parts 2 and 3 ) that exhibit position correlations supporting other modal connections. This straightforward analysis, however, is simply not available to the holist.
} 
(24) into a claim that delivers rigidity in all cases is to look at the only whole that is not a proper part of anything, namely, the mereologically maximal element, the universe.

In other words, holism** collapses into monism, the view that there is only one fundamental entity, and each part depends on that fundamental entity. ${ }^{41}$ As mentioned, some holists are indeed monists. However, one might want to endorse holism and yet deny the stronger monist view. Indeed, the holist intuition we are discussing here concerns the peculiar features of specific quantum systems, while monism as a general metaphysical thesis clearly depends upon important additional considerations. As a matter of fact, there are reasons for thinking that some interpretations of quantum mechanics are simply not amenable to a monistic rendering. ${ }^{42}$

Having said this, let us now assume that both structuralism and holism are not entirely satisfactory as ontological accounts of quantum composite systems, and turn to the question whether there is a better alternative. ${ }^{43}$

\footnotetext{
${ }^{41}$ It collapses in fact in a particular kind of monism, namely, Schaffer's version of priority monism, where the only fundamental entity and the less fundamental ones are mereologically related; see (Ismael and Schaffer [forthcoming]).

42 In particular, the monist who attempts to ground his/her view on quantum theory has to defend the claim that entanglement is a pervasive feature of the universe. But this claim is questionable. Schaffer himself, moreover, notes that monism is in immediate tension with some interpretations of quantum mechanics, such as Rovelli's relational interpretation. For a detailed assessment of monism in the quantum realm, see (Calosi [forthcoming]). It could be argued that the case for monism based on entanglement finds a better basis in quantum field theory. However, just how global and persistent entanglement is in the quantum-fieldtheoretic context is in fact open to debate. And, in any event, following the literature here we are discussing non-relativistic quantum mechanics only.

${ }^{43}$ One may claim that the problems for the holist are worse than those encountered by the structuralist, as the former cannot accommodate some kinds of entanglement, while the latter is 'just' forced to postulate generic notions of
} 


\section{Coherentism}

\subsection{Coherentism: The account}

Do structuralism and holism exhaust the metaphysical possibilities when it comes to accounting for the relations of dependence exhibited by entangled systems? Looking at the relevant literature, one might think that they do. However, we want to argue that there is another option: 'coherentism'.

Roughly, according to coherentism the right conclusion to draw from the empirical evidence involving quantum entangled systems is neither (i) that physical relations are prior to physical objects, nor (ii) that objects depend upon a more fundamental whole of which they are a part. Rather, what we should say is that objects may be regarded as fundamental, provided that an essential part of their being what they are is not taken to derive from 'lower' or 'upper' more fundamental levels in a hierarchy, and is instead conjectured to stem, so to put it, from 'horizontal', same-level structures of mutual relations of ontological dependence. In this context, coherentism takes from structuralism the emphasis on symmetric relations, and from holism the emphasis on 'global' ontological dependence between more or less traditional objects. The resulting idea is that the relevant physical facts are to be interpreted in terms of symmetric

dependence. While we are happy to concede this, we note that, on the other hand, structuralism is more revisionary, in that it makes relations more fundamental than relata (or at least equally fundamental). Furthermore, as we pointed out, both positions need independent arguments in favour of their preferred reading of the quantum state. Based on this, one may still claim that, all things considered, holism and structuralism are equally unsatisfactory. Thanks to an anonymous referee for pressing this point. 
relations of ontological dependence between certain groups of nonrelational entities.

To illustrate our proposal, it is useful to start from the structuralist insight that entangled particles are essentially related in some (generic) way. For present purposes, let us capture this via the following claim of necessary involvement:

$$
\square_{x x=\left\{x_{1}, x_{2}\right\}} R x_{1} x_{2}
$$

According to Equation (25), if two entangled entities exist, then there is a relation holding between them.

Now, as we have seen, structuralists take the relation to be physical, and to be a fundamental ontological item. Accordingly, they claim either that the two entities $x_{1}$ and $x_{2}$ are mere 'epiphenomena', since they are reducible to the relation; or, on a milder reading, that the relation necessitates the existence of two 'placeholders' with the specific features determined by the relation itself. In fact, structuralists claim that the physical relation plays a double role: on the one hand, it relates $x_{1}$ and $x_{2}$, as per Equation (25); on the other, it is one of the relata of the dependence relation tracked (in the present case) by entanglement, the other relatum being the 'plurality of' $x_{1}$ and $x_{2}$. This is exactly where structuralists and coherentists part ways. To appreciate this departure, consider the following revealing passage from Fine ([1995], pp. 282-4): 
It is sometimes thought that there are objects whose nature can be understood in terms of one another $[\ldots]$ in addition to possessing constitutive essential properties they will also enter into constitutive essential relations. Let us therefore say that an essential relationship between two or more objects is irreducible if it is not a logical consequence of the essential properties of the objects considered separately or of the essential relationships among disjoint proper subsets of the objects. Two objects may then be taken to be reciprocally dependent, under the present consequentialist conception, just in case they stand in an irreducible essential relationship, either together or in partnership with other objects.

What the coherentist does is maintain that in the entanglement case, the relevant physical systems exhibit essential collective features in Fine's sense.

This is crucial, as this sort of essentiality entails that the relevant facts about the particles considered together are irreducible, that is, they are neither a logical consequence of, nor do they supervene on, any disjoint proper subset of properties of the relata. In quantum jargon, this means that the probability distribution of the relevant degrees of freedom captured by $R$ does not factorize. This ensures that the coherentist analysis applies to cases of genuine entanglement, that is, to GMW-entanglement as defined in Section $2^{44}$

\footnotetext{
${ }^{44}$ As a matter fact, it applies non-trivially only to those cases. For, in the case of non-GMW entanglement, the probability distribution factorizes. Thus, the relevant facts do not qualify as essential to the components of the system considered together.
} 
Once the essentiality of the interconnection between the subsystems is recognized as a crucial aspect, the coherentist insists that this should not be interpreted in terms of some physical relation being more fundamental than its relata, but rather in terms of the two relata being mutually dependent, as Fine himself suggests. That is,

$$
\square_{x_{1}}\left(E x_{1} \rightarrow E x_{2}\right) \wedge \square_{x_{2}}\left(E x_{2} \rightarrow E x_{1}\right)
$$

Indeed, we take Equation (26) to be the core of metaphysical coherentism. It is, to repeat, the claim that entangled particles are mutually ontologically dependent-this being understood in the sense that physical objects are fundamental and at the same time constitutively dependent on each other. Note that this is not to say that the two particles are mutually dependent in general, as they may well exist unentangled. The claim is that once they interact in certain ways, they become symmetrically dependent on one another.

It is important to stress (again) that the relation of ontological dependence at stake here is one whereby the existence and qualitative profile of one entity depends on the existence and qualitative profile of the other and vice versa. This is crucial because, as we explained, a simple conditional statement to the effect that if $x$ exists so does y would be rather uninteresting and would not capture the essential features of entangled systems, especially in cases of GMW-entanglement. For, genuine entanglement primarily concerns the states of physical systems, hence their properties. Fortunately, it 
is by no means necessary to understand Equation (26) as a simple conditional involving existence. To the contrary, as pointed out earlier, we take expressions such as ' $x_{1}$ ' and ' $x_{2}$ ' to refer to two things that exist with certain properties. So understood, we contend, the essentiality of the necessary-involvement relation in Equation (25), and the mutual dependence relation in Equation (26) ground, and explain, the typical phenomena related to genuine entanglement, such as non-factorizability of states, non-local correlations etc.

Acknowledging the possibility and actuality of 'partnership connections', coherentism allows for 'horizontal webs' (or 'loops', or 'chains', or whatever conveys the idea that there are no vertical pyramids of any sort) of dependence, such as:

$$
\square_{x_{i}}\left(E x_{i} \rightarrow E x_{j}\right) \wedge \square_{x j}\left(E x_{j} \rightarrow E x_{k}\right) \wedge \square_{x_{k}}\left(E x_{k} \rightarrow E x_{i}\right)
$$

It should be clear by now that contrary to what one may think, this is not explanatorily deficient: we are not just saying ' $a$ depends on $b$ and $b$ depends on $a$ ', but rather something like ' $a, b, c, \ldots n$ ' and their properties collectively ground the whole or plurality $P$ with such and such properties (the entangled system), and the latter makes it possible for $a, b, c, \ldots, n$ to exist and/or to exemplify certain properties/relations'. In this sense, the relevant analogy here is with the 'sophisticated', or 'holistic' sort of coherentism about justification in epistemology: there, the claim is not that there is a linear dependence between entities (beliefs) so that, in the end, a belief 
justifies itself; rather, beliefs partially support each other by constituting a belief system that by virtue of having certain features, makes the constituting beliefs justified. In the words of BonJour ([1988], pp. 91-2), for instance:

It is at the local level of justification that inferential justification appears linear [...] However [...] a coherence theory will claim, the apparent circle of justification is not in fact vicious because it is not genuinely a circle: the justification of a particular empirical belief finally depends, not on other particular beliefs as the linear conception of justification would have it, but instead on the overall system and its coherence.

This also makes the distance from structuralism quite clear. Compare, for instance, with what Wolff ([2012], p. 610) says when contrasting physical structuralism with a particular version of mathematical structuralism:

(ODO) states a certain kind of holism: individual mathematical objects depend on all the other objects in their domain. This is in contrast to claims about ordinary objects, which we take to be largely independent of other ordinary objects [...] (ODO) by itself isn't a form of structuralism. (ODO) does not claim any priority of structure over objects, it asserts an interdependence of certain kinds of objects.

ODO is the claim that each object in the domain $D$ of some mathematical structure depends on every other object in $D$ (Linnebo 
[2008]). Something like this is, essentially, what we have in mind here.

It is easy to see that the view being put forward is not a holist view either. For, arguably, coherentism per se is compatible with the idea that in some cases, there is no whole to begin with (which is already relevant in view of the close connection between holism and monism highlighted earlier). Indeed, even though the mutual interdependence of objects is naturally expressed in terms of wholes being 'richer' than their separate parts, strictly speaking on a coherentist construal no whole needs to be posited as ontologically prior to its parts. ${ }^{45}$ Indeed, no whole needs to be posited at all in addition to the plurality of mutually related entities! $!^{46}$

The difference between coherentism on the one hand and structuralism and holism on the other can also be expressed in the following way: It may look as though coherentism has to start from some sort of structuralist reading of the quantum state, in that it claims that entangled parts are essentially related. However, we could start from a holist reading as well, and say that there is a monadic property that is instantiated by the alleged mereological fusion of the entangled particles, and that property necessitates a

\footnotetext{
${ }^{45}$ It is worth pointing out that while in an obvious sense a set of mutually dependent entities makes up a whole, different stories can be told concerning how wholes are determined, and the connection between the physical and the metaphysical aspect of composition. One may want to insist, for instance, that a physical composition process has indeed taken place whenever symmetric dependence relations are instantiated, and that the former is responsible for the existence of the latter. While we are sympathetic to this view, this is an additional claim.

${ }^{46}$ Of course, this depends on one's answer to the special composition question, and perhaps on one's opinion on composition as identity. But we do not need to get into details here.
} 
relation between the particles. For instance, in the simple case of the singlet state, it could be said that the monadic property 'having total spin $=0$ ' necessitates the relation 'having opposite spin' holding between individual subsystems. Alternatively, one may start from a pluralist ontology of individual physical systems and add irreducible physical relations (as per Teller's 'relational holism'; see Teller [1986]; Morganti [2009b]). This sort of 'indifference'-far from constituting a problem-indicates that coherentism constitutes a sui generis ontological view, genuinely different from extant alternatives. Any of these choices will do for the coherentist, because for her each of them is simply a particular way to get to what is really fundamental, symmetric dependence relations that set constraints, as it were, on their relata and their properties-the latter being, importantly, the only physical entities that have to be posited in the ontological inventory.

One last remark is in order. Given that structuralists and holists emphasise vertical dependence relations while coherentism focuses on horizontal 'webs' of mutual dependence, one could maintain that there is room for views that combine coherentism with either holism or structuralism or even both. While we have nothing to object to this, we note that: (i) given that these mixed views would entail greater complexity, it should be shown that they are required, or at any rate recommended, for explanatory purposes, for instance, because there is some specific explanatory work that coherentism cannot do, or does worse than structuralism and/or holism. If anything, we suggested in this article that this is not the case; (ii) at any rate, even 
if a mixed view turned out to be necessary (see remarks in next section), it seems coherentism would be part of it, which is already sufficient for our present purposes.

\subsection{Coherentism: The best of both worlds?}

We argued that coherentism strikes a nice balance between structuralism and holism: it incorporates insights from both, while steering clear of some controversial assumptions these two views need to make. In so doing, we think, coherentism gives us a picture of reality, from which several explanatory stories can be extracted, possibly going beyond the case of entanglement. Let us now look at this in more detail.

Since the present article is primarily concerned with the philosophical analysis of entanglement, one may first of all ask at this point for a detailed treatment of the specific difficulties that arise in the philosophy of physics when entangled systems are concerned. This primarily boils down, of course, to providing a detailed account of quantum correlations as they emerge in EPR-like settings, and of the well-known violations of Bell-type inequalities. Concerning this, to avoid misunderstanding, we first point out explicitly that our proposal is a squarely metaphysical one. As such, it cannot be expected to provide a solution to the problem represented by the empirically observed violations of Bell's inequalities, but rather to suggest a specific philosophical gloss on it-a gloss that, we claim, should be preferred over other metaphysical alternatives for the reasons considered in the article, having to do with explanatory power at a 
more general level. Indeed, whatever choices there may be available for dealing with the problems related to Bell's theorem, it is reasonable to expect that they are equally accessible to coherentists, structuralists and holists. Having said this, at any rate, here's a suggestion for analysing correlations violating Bell inequalities in coherentist terms.

First of all, it may be useful to recall the basic structure of Belltype arguments, which is the following: assuming that (i) measurements determine sharp values for the measured observables, (ii) values of the relevant spin-projections for the particles just before measurement are related to the initial state of the particles (when the entangled system is prepared), (iii) the successive outcomes of repeated identical measurements, in particular of the type to be performed on one of two entangled particles, are always genuinely random, (iv) any measurement result directly corresponds to an actually possessed property, and (v) locality holds, so that the outcomes of space-like related measurements must be independent, then certain inequalities follow that can be shown to be violated by at least some quantum composite systems (both at the abstract level of the theory's formalism and experimentally), that is, by genuinely entangled systems. Not surprisingly, coherentists can suggest that (v) has to go, and in particular that factorizability fails, and measurements on space-like related entangled particles are not independent, due to a failure of what is known as outcome independence. That is, for any two genuinely entangled and space-like related particles 1 and 2, 
measurement results $x$ and $y$, respectively, apparatus settings $i$ and $j$, respectively, and any eventual set of hidden variables $H$, it is not the case that $\mathrm{P}_{1}(x \mid i, j, y, H)=\mathrm{P}_{1}(x \mid i, j, H)$ and $\mathrm{P}_{2}(y \mid i, j, x, H)=\mathrm{P}_{2}(y \mid i$, $j, H){ }^{47}$ And the reason for this is that the qualitative profiles of the particles 1 and 2 are symmetrically dependent, hence the fact that outcome $x$ obtained at the 1-wing determines the outcome at the 2wing - which will be a $y$-outcome due to the system's initial state, the observed $x$-outcome and the relevant laws of nature. Again, we do not claim that this story is a particularly new one, or one that is only available to the coherentist. We note, however, the following: First of all, the existence of a symmetric dependence relation does provide (at least, of course, for those non-sceptical towards talk of ontological dependence) a non-causal explanation of the correlation in question. Compare this, for instance, with the causal analysis in (Ismael and Schaffer [forthcoming]): what we are suggesting is that by endorsing coherentism, one might invoke a failure of locality-in their terminology_that does not feature any causal component, contrary to what they claim. The same goes, it seems, when coherentism is contrasted with other putative explanations based on 'common causes'. Moreover, we note that the structuralist claim that entanglement is a genuine physical relation is tricky here: for, either this is taken literally, but then it seems in fact hard to provide an explanation, since presumably this type of relation must obey the usual constraints of finite-speed propagation of causal signals and, consequently, locality; alternatively, talk of entanglement being a

\footnotetext{
${ }^{47}$ Where $\mathrm{P}(x \mid y)$ stands, as usual, for the conditional probability of $x$ given $y$.
} 
physical relation is not taken literally, but then we don't see any other way of understanding this if not in coherentist terms. As for holism, something similar holds: for, presumably, the holist will say that the non-local correlation is explained in terms of facts about the twoparticle whole-say, total spin of zero plus the Pauli exclusion principle holding for its two components; but then again, either a story in terms of a physical link among the components is told, in which case these problems arise again, or one says that the whole is prior to the parts and the latter are therefore mutually ontologically related with respect to their qualitative profiles, in which case what really does the explaining here is just the coherentist claim of symmetric dependence.

Continuing now our comparative assessment, coherentism easily handles the cases we discussed in this article. For instance, in the case of entanglement distillation, the coherentist will simply say that dependence relations are confined-so to speak-to some proper parts of the composite system (if there is any), or between some members of the plurality of those parts (if there is no mereological fusion of those members), whereas they spread over all of its parts in the case of entanglement dilution. As for the difference between completely and non-completely entangled systems, consider the noncompletely entangled state in Equation (23). The coherentist account ${ }^{48}$ will yield that there are symmetric relations of dependence between 1 and 2 on the one hand and 3 and 4 on the other, and no other significant dependence relation, which is exactly what we were

\footnotetext{
${ }^{48} \mathrm{We}$ are restricting coherentism to the core claim in Equation (26) here.
} 
after. In the case of a $n$-particle completely entangled case, instead, it will deliver the following:

$$
\square_{x_{i}}\left(E x_{i} \rightarrow E x_{j}\right) \wedge \square_{x_{j}}\left(E x_{j} \rightarrow E x_{i}\right)
$$

for each $x_{i, j}$ among the $x x=\left\{x_{1}, \ldots, x_{n}\right\}$ with $i \neq j$. A similar story can be told, more generally, in all the cases in-between maximal, complete entanglement and complete non-entanglement (including the threeparticle case discussed in Footnote 40). In each of these cases, the coherentist will postulate mutual dependence relations holding only among those systems that are effectively entangled and, consequently, are not in states that attribute a complete, objective set of properties to them independently of the surrounding environment.

Generalizing from the case of entanglement, other issues a coherentist framework might shed light on include composition and Humean supervenience (see Calosi and Morganti [2016]), and structuralism in physics and ontic structural realism, including considerations having to do with particle-types and the role of group theory in quantum mechanics (see Morganti [unpublished]).

To the foregoing considerations related to explanatory power, one may wish to add 'a conservativeness argument', to the point that the account allows one to: (i) build an ontology of 'traditional' objects; and (ii) claim that objects can be mutually dependent when it comes to their qualitative profile, but also retain the traditional bottom-up dependence structure when it comes to mereological structure. 
Having now made our case for coherentism, let us consider some potential problems before closing. One objection is that coherentism not only allows for, but actually requires symmetric and cyclic relations of dependence, but this goes against the widely held assumption (see Lowe [2006]; Schaffer [2010]) that genuine metaphysical explanations must be based on asymmetric relations, otherwise a vicious circularity arises between explananda and explanantia. Is this a real problem? First of all, let us note that structuralists too maintain that neglecting symmetric relations of dependence might simply be due to a bias in favour of foundationalist metaphysical accounts, in particular mereological atomism. For example, here is McKenzie ([2014], p. 3; see also French [2010], pp. 102-3):

I appreciate that it is awkward to speak of reciprocated or 'symmetric' priority when the word 'priority' connotes asymmetry. The use of this term may be put down to a widespread bias against symmetric dependence relations-a bias I reject.

As a matter of fact, non-standard, non-foundationalist, and nonatomistic models of metaphysical structure are receiving increasing attention lately (see, for instance, some of the essays in Bliss and Priest [forthcoming]), so this cannot be a decisive argument against the account being put forward here.

Indeed, it seems obvious to us that general metaphysical theses should be sensitive to empirical input, and to an open-minded 
approach to the interpretation of science. Thus, if it emerges that non-hierarchical metaphysical structures manage, all things considered, to provide a satisfactory philosophical explanation of a certain domain of reality, it would appear simply wrong to discard them on the basis that a priori it is assumed that the relevant philosophical notions must have certain formal features, and reality has to follow suit. Our proposal here is exactly that one should not be misled and generalize intuitions that may hold (or at least appear very plausible) in the more limited domain of mereological relationsand perhaps only at the level of common sense and middle-sized dry goods. Indeed, in the case of quantum mechanical systems it might, all things considered, be best to think of them as characterized by 'horizontal' networks of dependence, different in important respects from both classical mereological structures and more revisionary ontological accounts currently on offer-which all obey the customary hierarchical view of reality. ${ }^{49}$

Lastly, there might be a worry about informativeness. The thought being that, exactly like structuralism, coherentism is in fact unable to deliver rigid claims of dependence. Consider two entangled particles $x_{1}$ and $x_{2}$. Isn't a generic claim to the point that particle $x_{1}$ depends on there being some particle that has a particular qualitative

\footnotetext{
${ }^{49}$ Besides quantum entanglement and the other scenarios mentioned in the course of the article, physics suggests other places where a metaphysical coherentist might want to look. Consider the law of quantum chromodynamics according to which there are no coloured particles, so that quarks necessarily come in groups of three. Doesn't this suggest that these particles are mutually existentially dependent? And of course one may want to explore the applicability of coherentism beyond physics.
} 
profile and stands in the appropriate relation to $x_{1}$ (and vice versa), all that we have?

While we think that generic dependence may have some role to play, we think the answer to this question is negative. Coherentism starts off recognizing that there is an essential relation between the entangled particles. It is true that the relation in question might have been a different one-that is, particles could have been entangled in another degree of freedom. It is also true that particle $x_{1}$ might have been entangled with particle $x_{3}$, rather than $x_{2}$. More generally, it is true that when one considers entanglement as characterizing a certain kind, class, family, or type of physical systems, all one can have is indeed generic dependence. Yet according to coherentism, once the relation $R$ is specified-that is, in concrete cases in which $x_{1}$ is entangled with $x_{2}-x_{1}$ 's qualitative profile with respect to the specific degree of freedom relevant for the case at hand depends precisely, or rigidly, on $\mathrm{x}_{2}$ and not some other particle (and vice versa). Note that this is true even if the two particles are entangled in more than one degree of freedom. Crucially, for the coherentist it is always specific entangled system-tokens that come first, in the order of explanation and at the metaphysical level. That is, while structuralists cannot go beyond generic dependence, coherentists obtain generic dependence, as it were, as a more or less obvious 'byproduct' of actual relations holding among specific objects, hence able to ground rigid dependencies. Given the widespread agreement, discussed earlier in the article, to the point that rigid claims are more informative, we thus conclude that coherentism provides an 
informative explanation of what it is at stake in cases of entanglement.

\section{Conclusion}

In this article, we presented a novel ontological account of entangled quantum systems, based on symmetric dependence relations between objects. We are confident that the account can (and should be) generalized. While the basic features of the view should, hopefully, be already clear, further work is of course required in order to make it complete and as explanatory as have suggested it can be. In the end, those made here were only the first steps towards coherentist quantum mechanics.

\section{Acknowledgments}

We would like to thank Fabrice Correia, Kit Fine, Mario Hubert, Kerry McKenzie, Emanuele Rossanese, and audiences in Geneva, Paris, and Rome for numerous suggestions on previous drafts of this article. We are particularly grateful to three anonymous referees of this journal for their insightful comments, that led to substantive revisions. Claudio Calosi acknowledges the generous support of the Swiss National Science Foundation (project numbers BSCGIo_157792 and 100012_165738).

Claudio Calosi

Department of Philosophy University of Geneva Geneva, Switzerland claudio.calosi@unige.ch 
Matteo Morganti Department of Philosophy, Communication, and Media Studies, University of Rome 3 Rome, Italy matteo.morganti@uniroma3.it

\section{References}

Bennett, C., Bernstein, H. J., Popescu, S. and Schumacher, B. [1996]: 'Concentrating Partial Entanglement by Local Operations', Physical Review A, 53, pp. 2046-52.

Bigaj, T. [2012]: 'Entanglement of $N$ distinguishable particles', Studies in Logic, Grammar, and Rhetoric, 27, pp. 25-35.

Bigaj, T. [2015]: 'Dissecting Weak Discernibility of Quanta', Studies in History and Philosophy of Modern Physics, 50, pp. 43-53.

Bliss, R. and Priest, G. [forthcoming]: Reality and Its Structure, Oxford: Oxford University Press.

Bohn, E. D. [2012]: 'Monism, Emergence, and Plural Logic', Erkenntnis, 76, pp. 211-23.

BonJour, L. [1988]: The Structure of Empirical Knowledge, Cambridge, MA: Harvard University Press.

Calosi, C. [2014]: 'Quantum Mechanics and Priority Monism', Synthese, 191, pp. 915-28.

Calosi, C. [2017]: 'On the Possibility of Submergence', Analysis, 77, pp. 501-11.

Calosi, C. [forthcoming]: 'Quantum Monism. An Assessment'. Philosophical Studies.

Calosi, C. and Morganti, M. [2016]: 'Humean Supervenience, Composition as Identity and Quantum Wholes', Erkenntnis, 81, pp. 1173-94.

Caulton, A. [2015]: 'Is Mereology Empirical? Composition for Fermions', in T. Bigaj and C. Wüthrich (eds), Metaphysics in Contemporary Physics, New York: Rodopi, pp. 293-322.

Correia, F. [2008]: 'Ontological Dependence', Philosophy Compass, 3, pp. 1013-32.

Dieks, D. and Lubberdink, A. [2011]: 'How Classical Particles Emerge from the Quantum World', Foundations of Physics, 41, pp. 1051-64.

Earman, J. [2015]: 'Some Puzzles and Unresolved Issues about Quantum Entanglement', Erkenntnis, 80, pp. 303-37.

Esfeld, M. and Lam, V. [2008]: 'Moderate Structural Realism about Space-Time', Synthese, 160, pp. 27-46. 
Esfeld, M. and Lam, V. [2011]: 'Ontic Structural Realism as a Metaphysics of Objects', in P. Bokulich and A. Bokulich (eds) Scientific Structuralism, Berlin: Springer, pp. 145-59.

Fine, K. [1995]: 'Ontological Dependence', Proceedings of the Aristotelian Society, 95, pp. 269-90.

French, S. [2010]: 'The Interdependence of Structure, Objects, and Dependence', Synthese, 175, pp. 89-109.

French, S. [2014]: The Structure of the World, Oxford: Oxford University Press.

Ghirardi, G., Marinatto, L. and Weber, T. [2002]: 'Entanglement and properties of composite quantum systems: a conceptual and mathematical analysis', Journal of Statistical Physics, 108, pp. 49-122.

Hasegawa, Y. [2012]: 'Entanglement between Degrees of Freedom in a Single-Particle System Revealed in Neutron Interferometry', Foundations of Physics, 42, pp. 29-45.

Healey, R. [2013]: 'Physical Composition', Studies in the History and Philosophy of Modern Physics, 44, pp. 48-62.

Horodecki, R., Horodecki, P., Horodecki, M. and Horodecki, K. [2009]: 'Quantum Entanglement', Review of Modern Physics, 81, pp. 865-942.

Ismael, J. and Schaffer, J. [forthcoming]: 'Quantum Holism: Nonseparability as Common Ground', Synthese.

Jaeger, G. [2010]: 'Individuation in Quantum Mechanics and Space-Time, Foundations of Physics', 40, pp.1396-409.

Ladyman, J. [2014]: 'Structural Realism', in E. N. Zalta (ed.), Stanford Encyclopedia of Philosoph, available at <plato.stanford.edu/archives/spr2014/entries/structuralrealism>.

Ladyman, J., Linnebo, O. and Bigaj, T. [2013]: 'Entanglement and Non-factorizability', Studies in History and Philosophy of Modern Physics 43, pp. 215-21.

Li, N. and Luo, S. [2008]: 'Classical states versus separable states', Physical Review A, 78, pp. 1-4.

Linnebo, O. [2008]: 'Structuralism and the Notion of Dependence', Philosophical Quarterly, 58, pp. 59-79.

Lowe, J. and Tahko, T. [2015]: 'Ontological Dependence', in E. N. Zalta (ed.), Stanford Encyclopedia of Philosophy, available at <plato.stanford.edu/archives/spr2015/entries/dependenceontological>. 
McKenzie, K. [2014]: 'Priority and Particle Physics: Ontic Structural Realism as a Priority Thesis', British Journal for the Philosophy of Science, 65, pp. 353-80.

McKenzie, K. [forthcoming]: 'Structuralism in the Idiom of Determination', British Journal for the Philosophy of Science.

Morganti, M. [2009a]: 'Ontological Priority, Fundamentality, and Monism', Dialectica, 63, pp. 271-88.

Morganti, M. [2009b]: 'Inherent Properties and Statistics with Individual Particles in Quantum Mechanics'. Studies in History and Philosophy of Modern Physics, 40, pp. 223-31.

Morganti, M. [unpublished]: 'From Ontic Structural Realism to Metaphysical Coherentism'.

Muller, F. A. and Saunders, S. [2008]: 'Discerning Fermions', British Journal for the Philosophy of Science, 59, pp. 499-548.

Muller, F. A. and Seevink, M. P. [2009]: 'Discerning Elementary Particles', Philosophy of Science, 76, pp. 179-200.

Pauli, P. [1925]: 'Uber den Zusammenhang des Abschlusses der Elektronengruppen im Atom mit der Komplexstruktur der Spektren', Zeitschrift für Physik, 31, pp. 765-83.

Saunders, S. [2006]: 'Are Quantum Particles Objects?', Analysis, 66, pp. 52-63.

Schaffer, J. [2010]: 'Monism: The Priority of the Whole', Philosophical Review, 119, pp. 31-76.

Schaffer, J. [2014]: 'Monism', in E. N. Zalta (ed.), Stanford Encyclopedia of Philosophy, available at <plato.stanford.edu/archives/spr2014/entries/monism/ >.

Teller, P. [1986]: 'Relational Holism and Quantum Mechanics', British Journal for the Philosophy of Science, 37, pp. 71-81.

Wang, H., Sun, L., Zang, S. and Yeon, K. [2012]: 'Scheme for Entanglement Concentration of Unknown Partially Entangled Three-Atom W States in Cavity QED', Quantum Information Process, 11, pp. 431-41.

Wilson, J. [2010]: 'What Is Hume's Dictum and Why Believe It?', Philosophy and Phenomenological Research, 80, pp. 595-637. Wolff, J. [2012]: 'Do Objects Depend on Structures?', British Journal for the Philosophy of Science, 63, pp. 607-25.

Zylstra, J. [forthcoming]: 'Collective Essence and Monotonicity'. Erkenntnis. 